\title{
Scorpion: Facilitating High Throughput Electron Microscopy
}

\author{
Venkata P. Dandey ${ }^{1}$, Peter Kahn ${ }^{2}$, Hui Wei ${ }^{1}$, Bridget Carragher ${ }^{1,3}$, Clinton S. Potter ${ }^{1,3}$ \\ 1. National Resource for Automated Molecular Microscopy, Simons Electron Microscopy Center, New \\ York Structural Biology Center, New York, NY, USA. \\ 2. Engineering Arts LLC, Tempe, AZ, USA. \\ 3. Department of Biochemistry and Molecular Biophysics, Columbia University, New York, NY, USA.
}

CryoEM has established itself as an essential technique for structural biology. Negative Stain EM is often the first step in optimizing samples to ascertain their suitability for subsequent vitrification and imaging on high-end, and very expensive, instruments. Current methods for negative stain screening and sample optimization are time consuming and tedious and consume far too much protein. Preparing girds for negative stain screening presents several challenges. Among these are highly non-uniform staining, stain artifacts, particle aggregation, and that the entire real estate of a grid is used for only one sample. The traditional method of preparing girds for negative stain consumes up to $3 \mu \mathrm{L}$ of sample and a different grid is used each time making for a very inefficient process as the grid needs to be transferred through the airlock of the TEM each time. Ideally, only a few $\mathrm{nL}$ of sample should be required for each negative stain evaluation and multiple independent samples should be imaged on a single grid. Our goal is to develop a system to automate these procedures and provide negative stain EM as an efficient and high throughput "assay" for structural biology optimization.

Here we demonstrate a robot, named Scorpion (Fig 1a), equipped with three piezo-electric based picoliter dispenser heads (Fig 1c) which can fire independently, that can be used to spot 50pl droplets of sample (Fig 1b), stain and water accurately and sequentially onto a single grid square of a 150mesh continuous carbon grid (Fig 2). Preliminary results show that this method can be used to achieve quite consistent results for negative staining protein samples.

We anticipate that the capabilities of Scorpion can be readily expanded to generate multiplexed samples on one TEM grid for fully automated small molecule structure determination using micro electron diffraction (MicroED). It has recently been demonstrated that obtaining atomic resolution structure of small molecules may be straightforward using MicroED [4]. It seems clear that sample preparation will rapidly become a bottleneck if this technique is widely adopted and one avenue for improving throughput is to put multiple samples onto a single grid. We will demonstrate these additional capabilities of Scorpion and discuss the details of using the instrument for this purpose [5].

\section{References:}

[1] Dandey et al., J Struct Biol. 202 (2018), p. 161.

[2] Razinkov et al., J Struct Biol. 195 (2016), p. 190.

[3] Wei et al., J Struct Biol. 202 (2018), p. 170.

[4] Jones CG et al., ACS Cent Sci. 4 (2018), p. 1587.

[5] (GM103310, OD019994-01) and the Agouron Institute (F00316). 

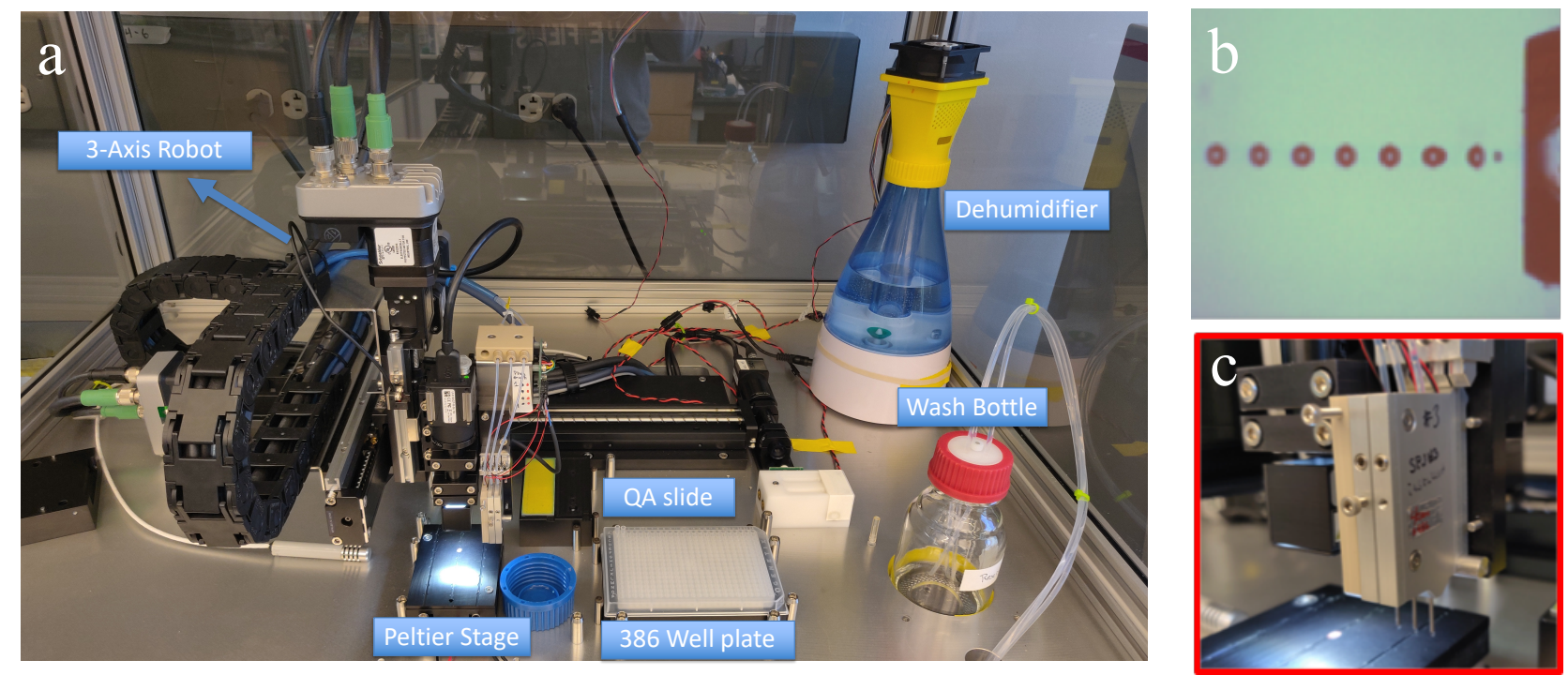

Figure 1. (a) Overview of Scorpion robot. (b) View of Cosine mode burst of 50pL droplets from piezo tip. (c) Multiple dispensers can sequentially dispense sample, stain and water.
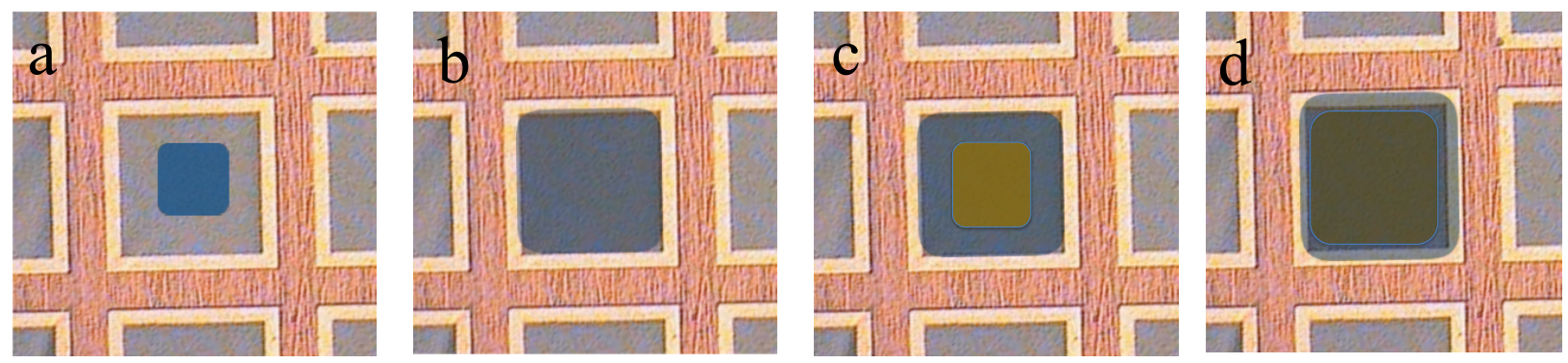

Figure 2. Schematic of negative staining using Scorpion. (a) 50pl of sample is dispensed at the center of grid square. (b) The sample spreads to a thin film following which, (c, d) stain is dispensed onto the same grid square and this can repeated if needed deeper stain.
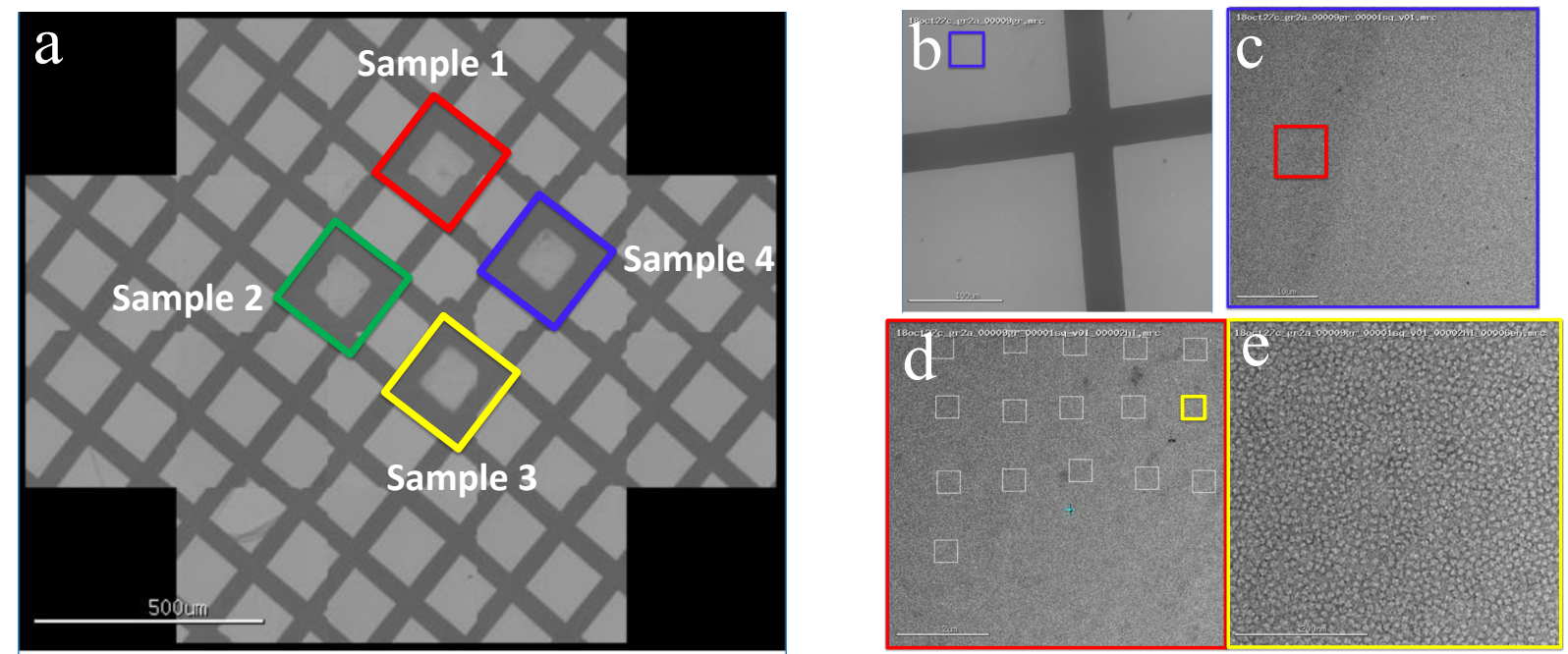

Figure 3. (a) Four samples dispensed onto four different squares on a 150mesh continuous carbon grid. (b, c, d, e) Sequential magnification images of one of the grid squares shows uniform sample and good stain across wide areas of the grid square. 